Pacific Journal of Mathematic 


\section{INVARIANT MANIFOLDS FOR REGULAR POINTS}

\section{JORGE LEWOWICZ}

In this article we prove, for a differentiable vector field or a diffeomorphism on a smooth manifold, that the set of points such that the semitrajectories issuing from them approach a particular semitrajectory at a given exponential rate, constitute a differentiable submanifold, provided the differential of the flow has a certain similar behavior on that trajectory. (See Theorem 1 below, for a precise statement). In particular, the stable manifold theorem for hyperbolic sets $([3],[6, X I])$ follows as a corollary.

Although we only consider the $C^{1}$-case, the same methods, which are essentially classical ([2, Ch. XIII]), could be applied to obtain higher differentiability properties.

Since I have not seen in the literature this type of results for points which are neither equillibrium nor periodic points, and on account of $[6, \mathrm{XI}-8]$, I thought that their publication might not be entirely devoid of interest.

1. Terminology and notation are standard. If $X$ is a differentiable vector field on a smooth manifold $M, \phi$ will always denote the corresponding flow, and $\phi_{t}$ the diffeomorphism $x \rightarrow \phi(x, t), x \in M, t \in R$. For brevity, we shall sometimes write $x(t)$ or $y(t)$ instead of $\phi(x, t)$ or $\phi(y, t)$ respectively.

Theorem 1. Let $M$ be compact smooth $\left(C^{\infty}\right)$ Riemannian manifold and $X$ a $C^{1}$-vector field. Assume that for some $x \in M$, there are subspaces $E, I ; E \oplus I=T_{x} M$, such that for some positive mumbers $K, \lambda, \mu, \mu<\lambda$, we have

$$
\left\|\phi_{s}^{\prime}(x(t)) e_{t}\right\|<K e^{-\lambda s}\left\|e_{t}\right\| \quad \text { for } \quad e_{t} \in \phi_{t}^{\prime}(x) E, s, t>0,
$$

and

$$
\left\|\phi_{-s}(x(t)) i_{t}\right\|<K e^{\mu s}\left\|i_{t}\right\| \quad \text { for } \quad i_{t} \in \phi_{t}^{\prime}(x) I, 0<s<t .
$$

Then, $W_{\lambda}(x)=\{y \in M / \overline{\lim }(1 / t) \log \operatorname{dist}(\phi(y, t), \phi(x, t))<-\lambda\} \quad$ is $a$ $C^{1}$-submanifold of $M$, such that $T_{x} W_{\lambda}(x)=E$.

Condition (1) means that $\phi_{t}^{\prime}$ strongly contracts the bundle $\mathrm{U}_{t>0} \phi_{t}^{\prime}(x) E$, while (2), which is equivalent to

$$
\left\|\phi_{s}^{\prime}(x(t)) i_{t}\right\| \geqq H e^{-\mu s}\left\|i_{t}\right\|, \quad t, s>0
$$

for some $H>0$, only says that $\phi_{t}^{\prime}$ does not contract as strongly on $\mathrm{U}_{t>0} \phi_{t}^{\prime}(x) I$. 
The following theorem may be proved applying Theorem 1 to the suspension of $M$. (See [1], Ch. 1.)

THEOREM 2. Let $M$ be a compact Riemannian smooth manifold and $f$ a $C^{1}$-diffeomorphism of $M$. Assume that there exists a point $x \in M$ and subspaces $E_{x}, I_{x}, E_{x} \oplus I_{x}=T_{x} M$ such that for some positive numbers $K, p, q, p<q<1$, we have

$$
\begin{gathered}
\left\|f^{m^{\prime}}\left(f^{n}(x)\right) e_{n}\right\|<K p^{n}\left\|e_{n}\right\|, \text { for } e_{n} \in f^{n^{\prime}}(x) E_{x}, \quad m, n>0 . \\
\left\|f^{-m^{\prime}}\left(f^{n}(x)\right) i_{n}\right\|<K q^{-m}\left\|i_{n}\right\|, \quad \text { for } \quad i_{n} \in f^{n^{\prime}}(x) I_{x}, \quad 0<m<n .
\end{gathered}
$$

Then $W_{p}(x)=\left\{y \in M / \varlimsup_{\overline{\lim }}(1 / n) \log \operatorname{dist}\left(f^{n}(y), f^{n}(x)\right)<-\log p\right\}$ is a $C^{1}$-submanifold of $M$, such that $T_{x}\left(W_{p}(x)\right)=E_{x}$.

Proof that Theorem 1 implies Theorem 2: Consider the suspension $\hat{M}$ of $M$, equipped with some Riemannian metric, and the corresponding vector field $X$. (We shall identify $M$ and $\pi(M \times\{0\}), \pi$ being the canonical projection of $M \times R$ onto $\widehat{M}$ ).

Since $X \neq 0$ on $M$, Theorem 1 may be applied to the semitrajectory $\phi(x, t), t>0$, taking $E_{x}$ as $E$, the subspace spanned by $I_{x}$ and $X(x)$ as $I$, and letting $-\log p,-\log q$ play, respectively, the roles of $\lambda$ and $\mu$. In this way, we get a $C^{1}$-submanifold $W_{\lambda}(x)$ of $M$; but if $y=\pi(y, s)$, and $s$ is not an integer, $\operatorname{dist}(\phi(y, t), \phi(x, t))$ is bounded away from zero for $t>0$. Thus, $W_{\lambda}(x) \subset M$, and this clearly implies $W_{\lambda}(x)=W_{p}(x)$. Since $T_{x} W_{\lambda}(x)=E_{x}$, this completes the proof.

If $x$ lies on a hyperbolic set ([3], [6]), its stable and unstable manifold may be obtained by a direct application of Theorem 2 (Theorem 1, if we were dealing with a vector field) to the diffeomorphisms $f$ and $f^{-1}$.

2. The results of this section will enable us to replace the manifold $M$ by an open subset of Euclidean space.

Let $M$ be a compact connected smooth submanifold of $R^{N}$ and let $r$ be the retraction $x \rightarrow r(x)$, where $r(x)$ is a point of $M$ with the property

$$
\|x-r(x)\|=\operatorname{dist}(x, M) .
$$

If the domain of $r$ is restricted to a suitable neighborhood $\Omega$ of $M$, then $r$ becomes a well defined smooth function (see [3]), such that $r(x)-x$ is orthogonal to $M$ for each $x \in \Omega$. Since for $x \in \Omega$, $r^{\prime}(x): R^{N} \rightarrow R^{N}$ is of maximal rank $n=\operatorname{dim} M$, and $r^{\prime}(x) v=0$ if $v$ is orthogonal to $T_{r(x)} M$, we have that for each $u \in T_{r(x)} M$ there is exactly one vector $v \in T_{r(x)} M$ such that $r^{\prime}(x) v=u$. 
If $X$ is a vector field on $M$ we may define a vector field $Y$ on $\Omega$ by letting $Y(x)$ be the unique vector of $T_{r(x)} M$ such that $r^{\prime}(x) Y(x)=$ $X(r(x))$. If $X \in C^{r}, r>1$, then, clearly, $Y \in C^{r}$; also $Y / M=X$.

Lemma 3. Let a be a real number and $Z^{a}$ the vector field defined on $\Omega$,

$$
Z^{a}=a(r(x)-x)+Y .
$$

Then, the normal bundle $N(M)$ of $M$ is invariant under the flow $\phi^{a}$ determined by $Z^{a}$ and

$$
\left\|\phi_{t}^{a^{\prime}}(x) \nu\right\|=e^{-a t}\|v\|
$$

for every $x \in M$ and $v \in N_{x}(M)$.

Proof. The invariance of $N(M)$ follows from the following relation:

$$
r^{\prime}(x) Z^{a}(x)=r^{\prime}(x) Y(x)=X(r(x))=Z^{a}(r(x)),
$$

which clearly implies that $r\left(\phi_{t}^{a^{\prime}}(x)\right)=\phi_{t}^{a}(r(x))$ for $x \in \Omega$.

The assertion concerning the norm of $\phi_{t}^{a^{\prime}}$ is a consequence of the following equalities, where we have written (, ) for the inner product in $R^{N}$ :

$$
\begin{aligned}
Z^{a}\left(\|r(x)-x\|^{2}\right) & =2\left((r(x)-x),\left(r^{\prime}(x) Z^{a}(x)-Z^{a}(x)\right)\right) \\
& =2\left((r(x)-x),\left(Z^{a}(r(x))-Z^{a}(x)\right)\right) \\
& =2((r(x)-x), X(r(x))-Y(x)-a(r(x)-x)) .
\end{aligned}
$$

Since $((r(x)-x), X(r(x))-Y(x))=0$, we have that $Z^{a}\left(\|r(x)-x\|^{2}\right)=$ $-2 a\|r(x)-x\|^{2}$. Therefore,

$$
\left\|\phi^{a}(x, t)-\phi^{a}(r(x), t)\right\|=e^{-a t}\|x-r(x)\|,
$$

which clearly implies the thesis.

Consider now a $C^{1}$-vector field $X$ on an open connected subset $\Omega$ of $R^{n}$, and a semitrajectory $\{\phi(x, t), t>0\}$ of $X$, whose closure is compact and contained in $\Omega$. Theorem 1 is a consequence of the following proposition.

Proposition 4. Assume that there are subspaces $E_{0}, I_{0}, E_{0} \oplus I_{0}=$ $R^{n}$, such that, writing $E_{t}\left(I_{t}\right)$ for $\phi_{t}^{\prime}(x) E_{0}$ (resp. $\left.\phi_{t}^{\prime}(x) I_{0}\right)$, we have

$$
\begin{aligned}
& \left\|\phi_{s}^{\prime}(x(t)) e_{t}\right\|<K e^{-\lambda s}\left\|e_{t}\right\|, \text { for } e_{t} \in E_{t}, t>0, s>0, \\
& \left\|\phi_{-s}^{\prime}(x(t)) i_{t}\right\|<K e^{\mu s}\left\|i_{t}\right\|, \text { for } i_{t} \in I_{t}, \quad 0<s<t,
\end{aligned}
$$


for some positive numbers, $K, \lambda, \mu, \mu<\lambda$.

Then $W_{\lambda}(x)=\left\{y \in \Omega / \varlimsup_{\lim }{ }_{t \rightarrow \infty}(1 / t) \log \|\phi(y, t)-\phi(x, t)\|<-\lambda\right\}$ is $a$ $C^{1}$-submanifold of $R^{n}$ tangent to $E_{0}$ at $x$.

Proof that Proposition 4 implies Theorem 1. We may assume that $M$ is embedded in, say, $R^{n}$. Extend the vector field $X$ to a neighborhood $\Omega$ of $M$ as in the previous lemma, choosing $a>\lambda$. Let $E_{0}$ be the subspace spanned by $E$ and $N_{x}(M)$ and take $I_{0}=I$; we may now apply Proposition 4 to get a $C^{1}$-submanifold $W_{\lambda}^{\prime}(x)$ of $R^{n}$. Then, $W_{\lambda}(x)=r\left(W_{\lambda}^{\prime}(x)\right.$ ), is a manifold (see [4], Lemma 3) and since $r^{\prime}(x) E_{0}=E$, the proof is complete.

3. In this section we prove two preliminary results.

Consider, as before, a $C^{1}$-vector field $X$ on an open connected subset $\Omega \subset R^{n}$, and a semitrajectory $\{\phi(x, t), t>0\}$ whose compact closure is included in $\Omega$. Let $E_{t}, I_{t}, t>0$ be as in Proposition 4, and call $P_{t}\left(Q_{t}\right)$ the projection of $R^{n}$ onto $E_{t}\left(I_{t}\right)$ along $I_{t}$ (resp. $\left.E_{t}\right)$.

Lemma 5. There is a positive number $M$, such that $\left\|P_{t}\right\|<M$, $\left\|Q_{t}\right\|<M, t>0$.

Proof. Suppose that $\left\|P_{t}\right\|$ is not bounded for $t>0$. Then we may find a sequence $t_{n} \rightarrow \infty$ and vectors $e_{t_{n}} \in E_{t_{n}}, i_{t_{n}} \in I_{t_{n}}, n=1,2, \cdots$ such that $\left\|e_{t_{n}}\right\| \rightarrow \infty$ and $\left\|e_{t_{n}}+i_{t_{n}}\right\|=1$. Moreover, we may assume that $\phi\left(x, t_{n}\right)$ converges to $y \in \Omega$, and that $\left(e_{t_{n}} /\left\|e_{t_{n}}\right\|\right)$ converges to some unit vector $u \in R^{n}$. Since $\left(-i_{t_{n}} /\left\|i_{t_{n}}\right\|\right)$ must also converge to $u$, we have that for $t>0,\left\|\phi_{t}^{\prime}(y) u\right\|<K e^{-\lambda t}$ and $\left\|\phi_{t}^{\prime}(y) u\right\|>H e^{-\mu t}$ (see $2^{\prime}$ ) in $\S 2)$ which is absurd. Inasmuch as $P_{t}+Q_{t}=I d, t>0$, this completes the proof.

The following technical lemma will be useful.

Lemma 6. Assume that $\phi(y, t)$ is defined in $\mid 0, b)$. Then, for $0<t<b$, we have

$$
\phi(y, t)-\phi(x, t)=\phi_{t}^{\prime}(x)(y-x)+\int_{0}^{t} \phi_{t-s}^{\prime}(x(s)) \Delta(x(s), y(s)) d s,
$$

where $A(x, y)=X(y)-X(x)-J(x)(y-x)$.

Proof. From

$$
\begin{gathered}
\frac{d}{d t}(\phi(y, t)-\phi(x, t))=X(\phi(y, t))-X(\phi(x, t)) \\
=J(x(t))(y(t)-x(t))+\Delta(x(t), y(t)),
\end{gathered}
$$


we get

$$
\begin{aligned}
& \phi_{-t}^{\prime}(x(t)) \frac{d}{d t}(y(t)-x(t))-\phi_{-t}^{\prime}(x(t)) J(x(t))(y(t)-x(t)) \\
& =\phi_{-t}^{\prime}(x(t)) \Delta(x(t), y(t)),
\end{aligned}
$$

which implies

$$
\frac{d}{d t}\left(\phi_{-t}^{\prime}(x(t))(y(t)-x(t))\right)=\phi_{-t}^{\prime}(x(t)) \Delta(x(t), y(t))
$$

since $\phi_{-t}^{\prime}(x(t)) \cdot \phi_{t}^{\prime}(x)=I d$ and $(d / d t) \phi_{t}^{\prime}(x)=J(x(t)) \phi_{t}^{\prime}(x)$ ([2], Ch. I). By integration we find

$$
\dot{\phi}_{-t}^{\prime}(x(t))(y(t)-x(t))=(y-x)+\int_{0}^{t} \phi_{-s}^{\prime}(x(s)) \Delta(x(s), y(s)) d s
$$

and applying $\phi_{t}^{\prime}(x)$ on the left we obtain the thesis of the lemma.

4. LEMma 7. Assume that $y(t), t>0$, is a semitrajectory of $X$ such that $\|y(t)-x(t)\|<\alpha e^{-r t}$, where $\alpha>0$ and $\mu<\gamma<\lambda$. Then $y(t)$ satisfies the integral equation

$$
\begin{aligned}
y(t)= & x(t)+\phi_{t}^{\prime}(x) P_{0}(y-x)+\int_{0}^{t} \phi_{t-s}^{\prime} P_{s} \Delta(x(s),(s)) d s \\
& -\int_{t}^{\infty} \phi_{t-s}^{\prime}(x(s)) Q_{s} \Delta(x(s), y(s)) d s .
\end{aligned}
$$

Proof. From Lemma 6 we get

$$
\begin{aligned}
y(t)-x(t)= & \phi_{t}^{\prime}(x) P_{0}(y-x)+\int_{0}^{t} \phi_{t-s}^{\prime}(x(s)) P_{s} \Delta(x(s), y(s)) d s \\
& +\phi_{t}^{\prime}(x)\left(Q_{0}(y-x)+\int_{0}^{t} \phi_{t-s}^{\prime}(x(s)) Q_{s} \Delta(x(s), y(s)) d s .\right.
\end{aligned}
$$

Since for large $s$,

$$
X(y(s))-X(x(s))=\int_{0}^{1} J((1-u) x(s)+u y(s)) d u(y(s)-x(s)),
$$

we have that $\|\Delta(x(s), y(s))\|<c\|y(s)-x(s)\|$ for some $c>0$; if $c$ is taken large enough, the same inequality holds for all $s>0$. Then, from the above formula we obtain, on account of (1), that

$$
\begin{aligned}
& \left\|\dot{\phi}_{t}^{\prime}(x)\left(Q_{0}(y-x)\right)+\int_{0}^{t} \phi_{-s}^{\prime}(x(s)) Q_{s} \Delta(x(s), y(s)) d s\right\| e^{\gamma t} \\
& <\alpha+K M e^{-(\lambda-\gamma) t}\|y-x\|+K M c \alpha e^{\gamma t} \int_{0}^{t} e^{-\lambda(t-s)} e^{-\gamma s} d s,
\end{aligned}
$$

which is bounded for $t>0$. By $\left(2^{\prime}\right)$ this implies the boundedness, 
for $t>0$, of

$$
\left\|Q_{0}(y-x)+\int_{0}^{t} \phi_{-s}^{\prime}(x(s)) Q_{s} \Delta(x(s), y(s)) d s\right\| e^{(\gamma-\mu) t} .
$$

Thus, $Q_{0}(y-x)=-\int_{0}^{\infty} \phi_{-s}^{\prime}(x(s)) Q_{s} \Delta(x(s), y(s)) d s$ as we had to show.

On the other hand it is important to notice that if $y(t), t \geqq 0$ is a continuous function with values in $\Omega$ that satisfies the integral equation

$$
\begin{aligned}
y(t)= & x(t)+\dot{\phi}_{t}^{\prime}(x) e_{0}+\int_{0}^{t} \phi_{t-s}^{\prime}(x(s)) P_{s} \Delta(x(s), y(s)) d s \\
& -\int_{t}^{\infty} \phi_{t-s}^{\prime}(x(s)) Q_{s} \Delta(x(s), y(s)) d s,
\end{aligned}
$$

$e_{0} \in E_{0}$, then $y(t)$ is also a trajectory of $X$ with $P_{0}(y(0)-x)=e_{0}$. In fact, since the differentiability of $y(t)$ follows by inspection of the right hand side of the equation, we may differentiate both sides to get

$$
\dot{y}(t)=\dot{x}(t)+J(x(t))(y(t)-x(t))+\Delta(x(t), y(t))=X(y(t)) .
$$

5. For each $\alpha>0$, and $\gamma, \mu<\gamma<\lambda$, let $y_{\alpha}(\gamma)$ be the space of continuous functions $t \rightarrow y(t), y(t) \in R^{n}, t \geqq 0$, such that $\|y(t)-x(t)\|<$ $\alpha e^{-\gamma^{t}}$. If $y, z \in y_{\alpha}(\gamma)$, let

$$
d(y, z)=\sup _{t>0}\|y(t)-z(t)\| e^{r t}
$$

it is not difficult to check, that with $d$ as the distance, $y_{\alpha}(\gamma)$ becomes a complete metric space.

Now for $e_{0} \in E_{0}$, consider the operator $T_{e_{0}}: y \rightarrow z$, where $y \in y_{\alpha}(\gamma)$ and $z: \mid 0, \infty) \rightarrow R^{n}$ is given by

$$
\begin{aligned}
z(t)= & x(t)+\phi_{t}^{\prime}(x) e_{0}+\int_{0}^{t} \phi_{t-s}^{\prime}(x(s)) P_{s} \Delta(x(s), y(s)) d s \\
& -\int_{t}^{\infty} \phi_{t-s}^{\prime}(x(s)) Q_{s} \Delta(x(s), y(s)) d s
\end{aligned}
$$

the fact that $\gamma>\mu$ ensures the convergence of the improper integral. Since for $y$ close to $x$

$$
\Delta(x, y)=\left(\int_{0}^{1}(J(1-u) x+u y)-J(x) d u\right)(y-x),
$$

the continuity of $J$ implies that for each $\varepsilon>0$, it is possible to choose $\alpha=\alpha(\varepsilon)>0$, such that if $\|y-x\|<\alpha$,

$$
\|\Delta(x, y)\|<\varepsilon\|y-x\| \text {. }
$$


For a given $\gamma, \mu<\gamma<\lambda$, choose $\varepsilon=\varepsilon(\gamma)$ such that $\varepsilon K M\left((\lambda-\gamma)^{-1}+\right.$ $\left.(\gamma-\mu)^{-1}\right)=1 / 2$, and let $\alpha(\gamma)$ or simply $\alpha$, be the corresponding $\alpha(\varepsilon(\gamma))$.

Lemma 8. For each $e_{0} \in E_{0}$ with $\left\|e_{0}\right\|<\alpha /(2 K), T_{e_{0}}$ is a contraction of $y_{\alpha}(\gamma)$.

Proof. We first show that for those $e_{0}, T_{e_{0}}: y_{\alpha}(\gamma) \rightarrow y_{\alpha}(\gamma)$.

Let $t \rightarrow y(t)$ belong to $y_{\alpha}(\gamma)$, and let $z=T_{e_{0}}(y)$; then, by (1) and (2), we have, for $t>0$,

$$
\begin{aligned}
\|z(t)-x(t)\| e^{\gamma t} \leqq & K e^{-(\lambda-\gamma) t}\left\|e_{0}\right\| \\
& +K M \in \alpha e^{-(\lambda-\gamma) t} \int_{0}^{t} e^{(\lambda-\gamma) s} d s+K M \varepsilon \alpha e^{(\gamma-\mu) t} \int_{t}^{\infty} e^{(\mu-\gamma) s} d s \\
< & K\left\|e_{0}\right\|+\alpha \varepsilon K M\left(\frac{1}{\lambda-\gamma}+\frac{1}{\mu-\gamma}\right) \leqq \alpha .
\end{aligned}
$$

On the other hand, if $y, \bar{y} \in y_{\alpha}(\gamma)$ and $z=T_{e_{0}}(y), \bar{z}=T_{e_{0}}(\bar{y})$, we have that

$$
\begin{aligned}
\|\bar{z}(t)-z(t)\| e^{\gamma t} \leqq & K M \varepsilon e^{-(\lambda-\gamma) t} \int_{0}^{t} d(y, \bar{y}) e^{(\lambda-\gamma) s} d s \\
& +K M \varepsilon e^{(\gamma-\mu) t} \int_{t}^{\infty} d(y, \bar{y}) e^{(\mu-\gamma) s} d s,
\end{aligned}
$$

for $t \geqq 0$, and consequently, $d(z, \bar{z})<(1 / 2) d(y, \bar{y})$. This completes the proof.

Thus, if $e_{0}$ is small enough, there is one and only one fixed point $y\left(t, e_{0}\right)$ of $T_{e_{0}}$ in $y_{\alpha}(\gamma)$, and on account of previous remarks, this fixed point is the unique semitrajectory of the vector field $X$, satisfying $P_{0}\left(y\left(0, e_{0}\right)-x\right)=e_{0}$ that belongs to $y_{\alpha}(\gamma)$.

Since the continuity in $e_{0}$ of $y\left(t, e_{0}\right)$ is an easy consequence of uniqueness, and $y\left(0, e_{0}\right)=y\left(0, e_{0}^{\prime}\right)$ implies readily $e_{0}=e_{0}^{\prime}$, we may state, letting $f=y\left(0, e_{0}\right)$ :

CoRollary 9. Let $B_{\alpha}=\left\{e_{0} \in E_{0} /\left\|e_{0}\right\|<\alpha / 2 K\right\}$. There is a continuous injective function $f: B_{\alpha} \rightarrow R^{n}$ with the following property: a semitrajectory of $X, \phi(y, t), t \geqq 0$, satisfies

$$
\|\phi(y, t)-x(t)\|<\alpha e^{-\gamma t}, t \geqq 0, \quad \text { and } \quad P_{0}(y-x)=e_{0} \in B_{\alpha},
$$

if and only if, $y=f\left(e_{0}\right)$.

6. Now we study the differentiability properties of $f\left(e_{0}\right)$ or 
$y\left(t, e_{0}\right)$. If the derivative of $y\left(t, e_{0}\right)$ in the direction of the unit vector $u \in E_{0}$ exists at $e_{0}$, and if we could differentiate under the integral sign, we would have that this derivative, $z_{u}\left(t, e_{0}\right)$, $\left\|e_{0}\right\|<\alpha /(2 K)$, satisfies:

$$
\begin{aligned}
z_{u}\left(t, e_{0}\right)= & \phi_{t}^{\prime}(x) u+\int_{0}^{t} \phi_{t-s}^{\prime}(x(s)) P_{s}\left(J\left(y\left(s, e_{0}\right)\right)-J(x(s))\right) z_{u}\left(s, e_{0}\right) d s \\
& -\int_{t}^{\infty} \phi_{t-s}^{\prime}(x(s)) Q_{s}\left(J\left(y\left(s, e_{0}\right)\right)-J(x(s))\right) z_{u}\left(s, e_{0}\right) d s .
\end{aligned}
$$

Let $V$ be the space of continuous functions $\left(t, e_{0}\right) \rightarrow z\left(t, e_{0}\right), t>0$, $\left\|e_{0}\right\|<\alpha / 2 K, z\left(t, e_{0}\right) \in R^{n}$, such that $\left\|z\left(t, e_{0}\right)\right\|<2 K e^{-\gamma t}$. With the distance $d$,

$$
d(z, \bar{z})=\sup _{\substack{t>0 \\\left\|e_{0}\right\|<\alpha / 2 K}}\left\|z\left(t, e_{0}\right)-\bar{z}\left(t, e_{0}\right)\right\| e^{\gamma t},
$$

$V$ is a complete metric space.

Lemma 10. For $z \in V$, define $T_{u}(z)=w$ by

$$
\begin{aligned}
w\left(t, e_{0}\right)= & \phi_{t}^{\prime}(x) u+\int_{0}^{t} \phi_{t-s}^{\prime}(x(s)) P_{s}\left(J\left(y\left(s, e_{0}\right)\right)-J(x(s))\right) z\left(s, e_{0}\right) d s \\
& -\int_{t}^{\infty} \phi_{t-s}^{\prime}(x(s)) Q_{s}\left(J\left(y\left(s, e_{0}\right)\right)-J(x(s))\right) z\left(s, e_{0}\right) d s .
\end{aligned}
$$

Then, for each $u \in E_{0},\|u\|=1, T_{u}$ is a contraction of $V$.

Proof. Since

$$
\begin{aligned}
\left\|w\left(t, e_{0}\right)\right\| \leqq & K e^{-\lambda t}+2 K^{2} M \varepsilon e^{-\lambda t} \int_{0}^{t} e^{(\lambda-\gamma) s} d s \\
& +2 K^{2} M \varepsilon e^{-\mu t} \int_{t}^{\infty} e^{(\mu-\gamma) s} d s \\
\leqq & 2 K e^{-\gamma t},
\end{aligned}
$$

$T_{u}$ maps $V$ into $V$. The fact that $T_{u}$ is a contraction follows at once from the inequality

$$
\begin{aligned}
\left\|w\left(t, e_{0}\right)-\bar{w}\left(t, e_{0}\right)\right\|< & K M \varepsilon e^{-\lambda t} \int_{0}^{t} e^{(\lambda-\gamma) s} d(z, \bar{z}) d s \\
& +K M \varepsilon e^{-\mu t} \int_{t}^{\infty} e^{(\mu-\gamma) s} d(z, \bar{z}) d s
\end{aligned}
$$

and the choice of $\varepsilon$.

Now, for $h \neq 0$, consider the quotient

$$
\begin{aligned}
q_{u}\left(h, t, e_{0}\right) & =\frac{1}{h}\left(y\left(t, e_{0}+h u\right)-y\left(t, e_{0}\right)\right) \\
& =\phi_{x}^{\prime}(t) u
\end{aligned}
$$




$$
\begin{aligned}
& +\int_{0}^{t} \phi_{t-s}^{\prime}(x(s)) P_{s} \frac{1}{h}\left(X\left(y\left(s, e_{0}+h u\right)\right)-X\left(y\left(s, e_{0}\right)\right)\right. \\
& \left.-J(x(s)) q_{u}\left(h, s, e_{0}\right)\right) d s \\
& -\int_{t}^{\infty} \phi_{t-s}^{\prime}(x(s)) Q_{s} \frac{1}{h}\left(X\left(y\left(s, e_{0}+h u\right)\right)-X\left(y\left(s, e_{0}\right)\right)\right. \\
& \left.-J(x(s)) q_{u}\left(h, s, e_{0}\right)\right) d s,
\end{aligned}
$$

and the difference

$$
\begin{aligned}
\delta_{u}\left(h, t, e_{0}\right)= & q_{u}\left(h, t, e_{0}\right)-z_{u}\left(t, e_{0}\right) \\
= & \int_{0}^{t} \phi_{t-s}^{\prime}(x(s)) P_{s}\left(J\left(y\left(s, e_{0}\right)\right)-J(x(s))\right) \delta_{u}\left(h, s, e_{0}\right) d s \\
& +\int_{0}^{t} \phi_{t-s}^{\prime}(x(s)) P_{s} D_{u}\left(h, s, e_{0}\right) d s \\
& -\int_{t}^{\infty} \phi_{t-s}^{\prime}(x(s)) Q_{s}\left(J\left(y\left(s, e_{0}\right)\right)-J(x(s))\right) \delta_{u}\left(h, s, e_{0}\right) d s \\
& -\int_{t}^{\infty} \phi_{t-s}^{\prime}(x(s)) Q_{s} D_{u}\left(h, s, e_{0}\right) d s,
\end{aligned}
$$

where

$$
D_{u}\left(h, s, e_{0}\right)=\frac{1}{h}\left(X\left(y\left(s, e_{0}+h u\right)\right)-X\left(y\left(s, e_{0}\right)\right)\right)-J\left(y\left(s, e_{0}\right)\right) q_{u}\left(h, s, e_{0}\right)
$$

Let $m(h)=\sup _{t>0}\left\|\delta_{u}\left(h, t, e_{0}\right)\right\| e^{\gamma t}, h \neq 0$; then, since

$$
\|q(h)\| \leqq(m(h)+2 K) e^{-r t}
$$

from the last equation we get, on account of

$$
\begin{aligned}
& \left\|D_{u}\left(h, t, e_{0}\right)\right\| \\
& \quad \leqq\left\|\int_{0}^{1} J\left((1-r) y\left(t, e_{0}\right)+r y\left(t, e_{0}+h u\right)\right) d r-J\left(y\left(t, e_{0}\right)\right)\right\|\left\|q_{u}(h, t, e)\right\|,
\end{aligned}
$$

that

$$
\begin{aligned}
\left\|\delta_{u}\left(h, t, e_{0}\right)\right\| e^{\gamma t} \leqq & \frac{K M \varepsilon m(h)}{\lambda-\gamma}+\frac{K M \rho(h)}{\lambda-\gamma}(m(h)+2 K) \\
& +\frac{K M \varepsilon m(h)}{\gamma-\mu}+\frac{K M \rho(h)}{\gamma-\mu}(m(h)+2 K),
\end{aligned}
$$

where

$$
\rho(h)=\sup _{t \geq 0}\left\|\int_{0}^{1} d r J\left((1-r) y\left(t, e_{0}\right)\right)+r\left(y\left(t, e_{0}+h u\right)\right)-J\left(y\left(t, e_{0}\right)\right)\right\| .
$$

Because of the choice of $\varepsilon$, we may write the last inequality, as 
$\left(\frac{1}{2}-K M\left(\frac{1}{\lambda-\gamma}+\frac{1}{\gamma-\mu}\right) \rho(h)\right) m(h) \leqq 2 K^{2} M\left(\frac{1}{\lambda-\gamma}+\frac{1}{\gamma-\mu}\right) \rho(h)$.

Since $\lim _{h \rightarrow 0} \rho(h)=0$, we get that $\lim _{h \rightarrow 0} m(h)=0$.

This shows that the derivative of $y\left(t, e_{0}\right)$ in the $u$ direction is the continuous function $z_{u}\left(t, e_{0}\right)$. In particular, it follows that $f$ (see Corollary 9) is a $C^{1}$-function.

CoROLlaRY 11. Let $B_{\alpha, t_{0}}=\left\{e_{t_{0}} \in E_{t_{0}} /\left\|e_{t_{0}}\right\| \leqq \alpha /(2 K)\right\} . \quad$ For each $t_{0} \geqq 0$ there is a continuously differentiable injective function $f_{t_{0}}: B_{\alpha, t_{0}} \rightarrow R^{n}$ with the following property: a semitrajectory of $X, \phi(y, t), t>0$, satisfies $\left\|\phi(y, t)-x\left(t_{0}+t\right)\right\|<\alpha e^{-\gamma t}$ for $t>0$, and $P_{t_{0}}\left(y-x\left(t_{0}\right)\right)=e_{t_{0}} \in B_{\alpha, t_{0}}$, if and only if, $y=f_{t_{0}}\left(e_{t_{0}}\right)$. Furthermore, $f_{t_{0}}^{\prime}(0) u=u, u \in E_{t_{0}}$.

Proof. It is clear that we would have obtained the same results if we had started from any semitrajectory $\phi\left(x\left(t_{0}\right), t\right), t \geqq 0, t_{0} \geqq 0$. Moreover, it is easy to check that, for a fixed $\gamma$, the constants $\varepsilon(\gamma)$ and $\alpha(\gamma)$ that we have chosen for the semitrajectory $x(t), t \geqq 0$, are also adequate for the semitrajectories $\phi\left(x\left(t_{0}\right), t\right), t \geqq 0, t_{0} \geqq 0$. So, with the exception of the last one, all the assertions of the corollary are a consequence of previous arguments. The last statement follows by inspection of the integral equation satisfied by $z_{u}\left(t, e_{t_{0}}\right)$ in the case $e_{t_{0}}=0$.

7. Lemma 12. Assume that for some $L>0$ and some $\gamma, \mu<$ $\gamma<\lambda,\|\phi(y, t)-x(t)\| \leqq L e^{-\gamma t}, t \geqq 0$. Then $y \in W_{\lambda}(x)$.

Proof. Let $\gamma^{\prime}$ be a number greater than $\gamma$ and less than, but close enough to $\lambda$. We may assume that $\alpha\left(\gamma^{\prime}\right)<\alpha(\gamma)$; take $t_{0}>0$ such that

$$
L e^{-\gamma t_{0}}<\alpha\left(\gamma^{\prime}\right) ; \quad L e^{-\gamma t_{0}}<\frac{M \alpha\left(\gamma^{\prime}\right)}{2 K}
$$

and observe that as a consequence of the last inequality, there is a point $z \in R^{n}$, such that

$$
\left\|\phi(z, t)-x\left(t_{0}+t\right)\right\|<\alpha\left(\gamma^{\prime}\right) e^{-r^{\prime} t},
$$

for $t \geqq 0$ and $P_{t_{0}}\left(z-x\left(t_{0}\right)\right)=P_{t_{0}}\left(\phi\left(y, t_{0}\right)-x\left(t_{0}\right)\right)$.

As both, $\left\|\phi(z, t)-x\left(t_{0}+t\right)\right\|$ and $\left\|\phi\left(y, t_{0}+t\right)-x\left(t_{0}+t\right)\right\|$ are less than $\alpha(\gamma) e^{-\gamma t}$ we must have $\phi(z, t)=\phi\left(\phi\left(y, t_{0}\right), t\right)$ for $t \geqq 0$, which implies $\|\phi(y, t)-x(t)\| \leqq N e^{-\gamma^{\prime} t}, t \geqq 0$, for some $N>0$.

Since $\gamma^{\prime}$ may be chosen arbitrarily close to $\lambda$, this completes the proof. 
Proof of Proposition 4. Let $y \in W_{\lambda}(x)$; we have that for some $L>0$, and some $\gamma, \mu<\gamma<\lambda,\|\phi(y, t)-x(t)\| \leqq L e^{-r t}$, if $t \geqq 0$. Take a $t_{0}>0$ such that $L e^{-\gamma t_{0}}<\alpha(\gamma), L e^{-\gamma t_{0}}<M(2 K)^{-1} \alpha(\gamma)$. Then $\phi_{-t_{0}} \circ f_{t_{0}}$ : $B_{\alpha, t_{0}} \rightarrow R^{n}$ is an injective $C^{1}$-function such that its range contains $y$ and, by the previous lemma, it lies on $W_{\lambda}(x)$. Define the topology of $W_{\lambda}(x)$ making $\phi_{-t_{0}} \circ f_{t_{0}}$ to be a homeomorphism onto a neighborhood of $y$ in $W_{\lambda}(x)$. The $C^{1}$-compatibility of the atlas constructed in this way is a consequence of Corollary 11 and the differentiability properties of the flow. The assertion concerning the tangent space to $W_{\lambda}(x)$ at $x$ also follows from the corollary.

\section{REFERENCES}

1. V. Arnold e V. Avez, Problémes Ergodiques de la Mechánique Classique, GauthierVillars, Paris, 1967.

2. E. Coddington and N. Levinson, Theory of Ordinary Differential Equations, McGraw-Hill, New York, 1955.

3. M. Hirsch and C. Pugh, Stable Manifolds and hyperbolic sets, Proceedings of Symposia in pure mathematics XIV, American Math. Soc.

4. J. Lewowicz, Stability Properties of a class of attractors, Trans. Amer. Math. Soc., 185, 1973.

5. J. Milnor, Topology from the differentiable viewpoint, University Press of Virginia, Charlottesville, 1965.

6. J. Palis, Seminario de Sistemas Dinámicos, IMPA, Rio de Janeiro, 1971.

UNIVERSIDAD Simon Bolivar

Apartado Postal 80659

Caracas, Venezuela 



\section{PACIFIC JOURNAL OF MATHEMATICS}

\section{EDITORS}

DONALD BABBITT (Managing Editor)

University of California

Los Angeles, California 90024

Hugo RossI

University of Utah

Salt Lake City, UT 84112

C. C. MOORE and ANDREW OGG

University of California

Berkeley, CA 94720

\section{J. DugundJI}

Department of Mathematics University of Southern California Los Angeles, California 90007

R. FinN and J. Milgram Stanford University Stanford, California 94305

\section{ASSOCIATE EDITORS}
R. ARENS
E. F. BECKENBACH
B. H. Neumann
F. WOLF
K. YoshidA

\section{SUPPORTING INSTITUTIONS}

UNIVERSITY OF ARIZONA

UNIVERSITY OF BRITISH COLUMBIA

CALIFORNIA INSTITUTE OF TECHNOLOGY

UNIVERSITY OF CALIFORNIA

MONTANA STATE UNIVERSITY

UNIVERSITY OF NEVADA, RENO

NEW MEXICO STATE UNIVERSITY

OREGON STATE UNIVERSITY
UNIVERSITY OF OREGON

UNIVERSITY OF SOUTHERN CALIFORNIA

STANFORD UNIVERSITY

UNIVERSITY OF HAWAII

UNIVERSITY OF TOKYO

UNIVERSITY OF UTAH

WASHINGTON STATE UNIVERSITY

UNIVERSITY OF WASHINGTON 


\section{Pacific Journal of Mathematics}

\section{Vol. 96, No. $1 \quad$ November, 1981}

Hédi Amara, Groupe des classes et unité fondamentale des extensions quadratiques relatives à un corps quadratique imaginaire principal $\ldots \ldots \ldots 1$

Douglas S. Bridges, On the isolation of zeroes of an analytic function $\ldots \ldots 13$ Andrew J. Casson and John L. Harer, Some homology lens spaces which

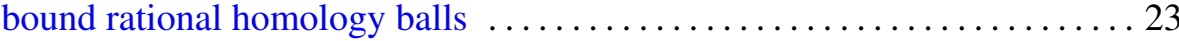

Z. A. Chanturia, On the absolute convergence of Fourier series of the classes $H^{\omega} \cap V[v]$

J.-F. Colombeau and Mário Carvalho Matos, On some spaces of entire functions defined on infinite-dimensional spaces $\ldots \ldots \ldots \ldots \ldots \ldots \ldots 63$

Edwin Duda, Pointwise periodic homeomorphisms on chainable continua . . .77

Richard F. Gustafson, A simple genus one knot with incompressible spanning surfaces of arbitrarily high genus $\ldots \ldots \ldots \ldots \ldots \ldots \ldots \ldots 1$

Fumio Hiai, Masanori Ohya and Makoto Tsukada, Sufficiency, KMS condition and relative entropy in von Neumann algebras

Ted Hurley, Intersections of terms of polycentral series of free groups and free Lie algebras. II .................................. 111

Robert Edward Jamison, II, Partition numbers for trees and ordered sets . . 115 R. D. Ketkar and N. Vanaja, A note on FR-perfect modules ............. 141 Michihiko Kikkawa, On Killing-Ricci forms of Lie triple algebras ....... 153

Jorge Lewowicz, Invariant manifolds for regular points 163

Richard W. Marsh, William H. Mills, Robert L. Ward, Howard Rumsey and Lloyd Richard Welch, Round trinomials .....

Claude Schochet, Topological methods for $C^{*}$-algebras. I. Spectral sequences

Yong Sian So, Polynomial near-fields?

Douglas Wayne Townsend, Imaginary values of meromorphic functions in the disk

Kiyoshi Watanabe, Coverings of a projective algebraic manifold .. 243

Martin Michael Zuckerman, Choosing $l$-element subsets of $n$-element sets 